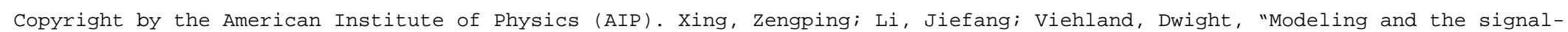

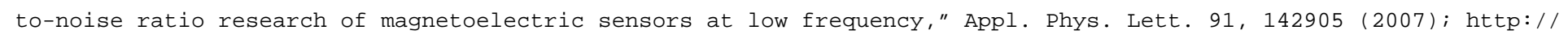
dx.doi.org/10.1063/1.2794435

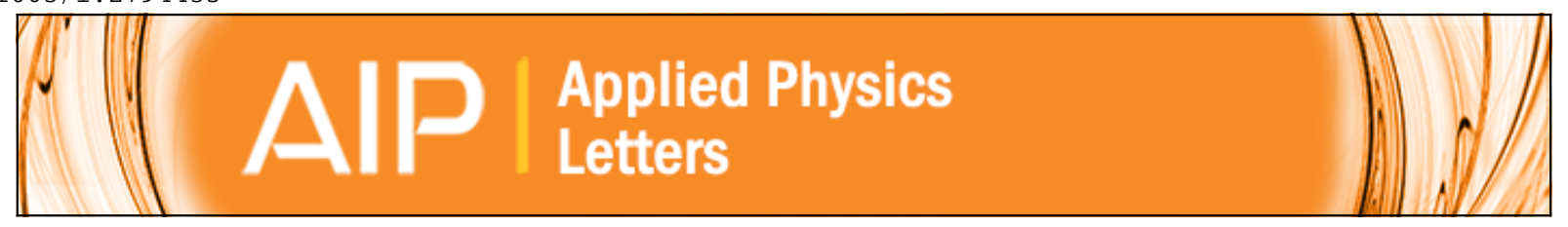

Modeling and the signal-to-noise ratio research of magnetoelectric sensors at low frequency

Zengping Xing, Jiefang Li, and Dwight Viehland

Citation: Applied Physics Letters 91, 142905 (2007); doi: 10.1063/1.2794435

View online: http://dx.doi.org/10.1063/1.2794435

View Table of Contents: http://scitation.aip.org/content/aip/journal/apl/91/14?ver=pdfcov

Published by the AIP Publishing

Over 700 papers \&

presentations on

multiphysics simulation wew now

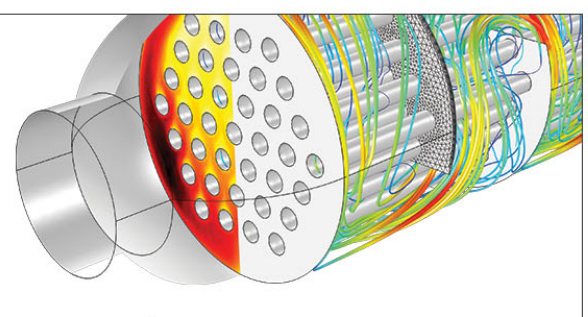

v СロMSロL 


\title{
Modeling and the signal-to-noise ratio research of magnetoelectric sensors at low frequency
}

\author{
Zengping Xing, ${ }^{\text {a) }}$ Jiefang Li, and Dwight Viehland \\ Materials Science and Engineering, Virginia Tech, Virginia 24061, USA
}

(Received 18 August 2007; accepted 16 September 2007; published online 2 October 2007)

\begin{abstract}
The authors have modeled magnetoelectric (ME) laminate sensor units by considering both the ME laminate and detection circuitry. Theory predicts and experiments confirm that the signal-to-noise ratio increases with increasing frequency in the low frequency range. Our findings provide useful insights into ME sensitivity estimation and "real-world" low frequency magnetic anomaly detection by ME sensors. (C) 2007 American Institute of Physics. [DOI: 10.1063/1.2794435]
\end{abstract}

The magnetoelectric (ME) effect-which is a magnetization induced by an electric field or a polarization induced by a magnetic field-has recently been of interest due to potential applications in magnetic sensors. ${ }^{1,2}$ The ME effect in two phase (magnetostrictive and piezoelectric) laminate composites is known to be much higher than that in single phase systems, particle composites, and thin films. ${ }^{2-7}$

To date, a parameter defined as $\alpha_{\mathrm{ME}}=\delta E / \delta H$ $=\delta(V / t) / \delta H$ (where $H$ is an applied magnetic field, $E$ and $V$ are the electric field and voltage induced across the piezoelectric layer, respectively, and $t$ is the thickness of the piezophase of the ME laminate) has been used as a figure of merit for ME sensors. ${ }^{8}$ It is an important parameter, which can relate the ME response of various types of ME laminate configurations; however, it is insufficient for understanding how a ME sensor performs in a real-world environment, where the signal-to-noise ratio (SNR) is most important. Here, in this letter, we develop an equivalent model for $\mathrm{ME}$ laminate sensor units operating at low frequencies. We confirm the predictions for ME direct measurement and SNR by experiments.

At low frequencies, where the wavelength is much longer than that of the composite dimensions, the laminate composite can be considered as a homogeneous body with effective parameters. Prior studies have solved for transverse and longitudinal modes in the free and clamped conditions. ${ }^{9}$ However, real-world boundary conditions can be very complicated, and the exact form of the solution might not be necessary. Thus, we approximate real-world conditions by using a quasiconstitutive linear equation that considers a "ME sensor system," given as

$$
{ }^{s} D_{k}={ }^{s} d_{k i}{ }^{s} T_{i}+{ }^{s} \varepsilon_{k n}{ }^{s} E_{n}+{ }^{s} m_{k n} H_{n} ;
$$

where ${ }^{s} T_{j},{ }^{s} E_{k},{ }^{s} D_{k}, H_{n},{ }^{s} d_{k i},{ }^{s} \varepsilon_{k i}$, and ${ }^{s} m_{k n}$ are the effective stress, electric field, dielectric displacement, magnetic field, piezoelectric coefficient, permittivity, and permeability of the ME sensor system.

To understand real-word ME sensor detection units with Eq. (1), a simple equivalent model might be helpful, as we can then model the electrical parameters of sensor and detection circuitry lumped together. If we consider such an approximation, then, in the absence of mechanical stress and magnetic field, Eq. (1) simplifies to

$$
{ }^{s} D_{k}={ }^{s} \varepsilon_{k n}{ }^{s} E_{n} .
$$

The effective permittivity of the ME laminate is a complex matrix, whose real and imaginary components are both frequency dependent. The complex permittivity is given by

$$
{ }^{s} \varepsilon_{k n}^{*}(\omega)={ }^{s} \varepsilon_{k n}^{1}(\omega)-j^{s} \varepsilon_{k n}^{2}(\omega)={ }^{s} \varepsilon_{k n}^{o}(\omega) e^{-j \theta} ;
$$

where $\omega$ is the angular frequency in $\mathrm{rad} / \mathrm{s},{ }^{s} \varepsilon_{k n}^{1}(\omega)$ and ${ }^{s} \varepsilon_{k n}^{2}(\omega)$ are the real and imaginary parts of the ${ }^{s} \varepsilon_{k n}^{*}(\omega)$ matrix. Since ${ }^{s} \varepsilon_{k n}^{o}(\omega)$ is the modulus of ${ }^{s} \varepsilon_{k n}^{*}(\omega)$ and $\tan \theta$ $={ }^{s} \varepsilon_{k n}^{2}(\omega) /{ }^{s} \varepsilon_{k n}^{1}(\omega)$, we can see that phase angle $\theta$ between the $E$ and $D$ vectors is also frequency dependent.

Since $D$ and the output charge $Q$ are related by $Q$ $=D \cdot A$ (where $A$ is the electrode's effective surface area), the current $I(t)$ produced by the piezoelectric phase can be acquired by the differential of the charge $Q$ in the time domain,

$$
I(t)=\frac{d Q(t)}{d t} .
$$

We can use the Laplace operation $L(\sim)$ to transform this into an equation in terms of the complex frequency $s=\sigma+j \omega$ (where $\sigma$ is the Neper frequency, and $\omega$ the angular frequency) as follows:

$$
I(s)=L(I(t))=L\left(\frac{d Q(t)}{d t}\right)=s Q(s)-Q_{0} ;
$$

where $Q_{0}$ is a constant. Assuming a sinusoidal excitation, we can replace $s$ with $j \omega$ and set the initial condition to make $Q_{0}=0$. Thus, Eq. (5a) can be rewritten as

$$
I(j \omega)=j \omega \cdot Q(j \omega) .
$$

We can then acquire the admittance $G$ of the ME sensor system by using Eqs. (2), (3), and (5b) as follows:

$$
\begin{aligned}
G & =\frac{I}{V}=\frac{I}{E \cdot t}=\frac{I \cdot \varepsilon_{0} \cdot e^{-j \theta}}{D \cdot t}=\frac{I \cdot A \cdot \varepsilon_{0} \cdot e^{-j \theta}}{Q \cdot t} \\
& =\frac{A \cdot \omega \cdot \varepsilon_{0}}{t}(\sin \theta+j \cos \theta) ;
\end{aligned}
$$

where $A$ is the effective electrode area of the ME sensor. A parallel equivalent model can then be set up following Eq. (6), as illustrated in Fig. 1(a) where $R=t / A \cdot \omega \cdot \varepsilon_{0} \sin \theta$ and $C=A \varepsilon_{0} \cos \theta / t$. 


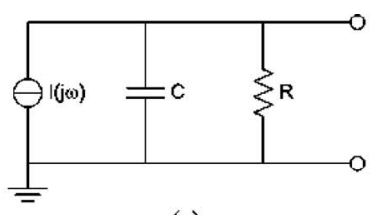

(a)

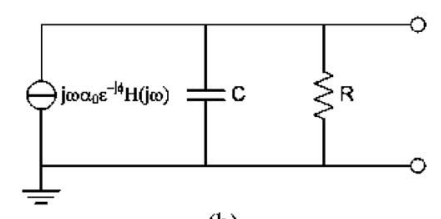

(b)

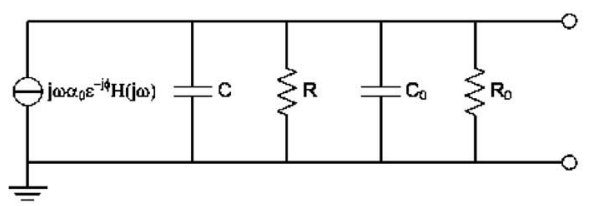

(c)

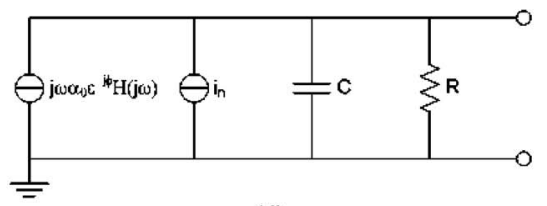

(d)

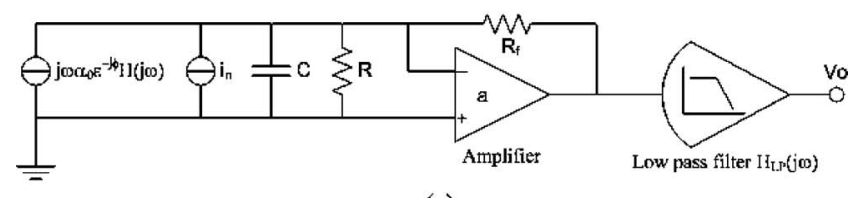

(e)

FIG. 1. Equivalent model for ME laminate sensor and detection method: (a) fundamental ME equivalent model; (b) ME laminate signal model; (c) equivalent circuitry for ME laminate with direct measurement method; (d) ME laminate model with noise consideration; and (e) ME laminate with noise spectra and current amplifier.

Next, we can define a fundamental parameter, named the ME charge coefficient $\alpha_{\mathrm{ME}}$, as $\alpha_{\mathrm{ME}}^{*}=\delta Q / \delta H$. This fundamental parameter is also complex

$$
\alpha_{\mathrm{ME}}^{*}(\omega)=\alpha_{1}(\omega)-j \alpha_{2}(\omega)=\alpha_{0}(\omega) e^{-j \phi} ;
$$

where $\alpha_{1}(\omega)$ and $-\alpha_{2}(\omega)$ are the real and imaginary parts, $\alpha_{0}(\omega)$ is the modulus of $\alpha_{\mathrm{ME}}^{*}(\omega)$, and $\tan \phi=\alpha_{2}(\omega) / \alpha_{1}(\omega)$. The angle $\phi$ is the phase delay of $Q$ in response to $H$, which is also frequency dependent. Since

$$
Q(t)=\alpha_{\mathrm{ME}}^{*}(\omega) H(t)=\alpha_{0}(\omega) e^{-j \phi} H(t),
$$

we can then rewrite Eq. (5b) as

$$
I(j \omega)=\alpha_{0}(\omega) \cdot e^{-j \phi} \cdot j \omega \cdot H(j \omega)=j \omega \cdot \alpha^{\prime} \cdot H(j \omega) .
$$

By combining Eq. (9) with Fig. 1(a), we can now set up a modified model for the ME sensor, as given in Fig. 1(b). This is a simple ME sensor signal model. Since at high frequency the stray capacitor, resistor, and inductor become important, significant error will be introduced by the model, thus, it is only suitable for the case of low frequencies. This model can be used to explain the experimental data for the case of ME direct measurement, where the output voltage rolls off with decreasing frequency at low frequencies. In this case, the equivalent model is shown in Fig. 1(c), and the detected voltage is given by
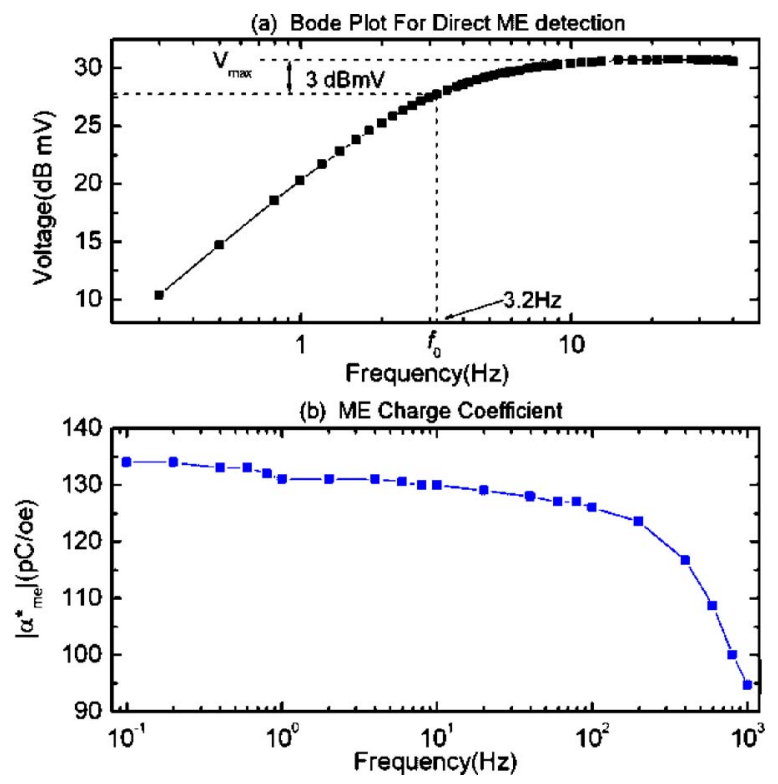

FIG. 2. (Color online) (a) Voltage as a function of frequency in ME direct measurement, illustrating a cutoff frequency below which shows that significant roll-off occurs with decreasing frequency, and (b) the magnetoelectric charge coefficient as a function of frequency, which shows a near constant value at low frequencies.

$$
V_{0}=I(j \omega) \cdot Z=\frac{j \omega \cdot \alpha^{\prime} \cdot R^{\prime}}{1+j \omega \cdot R^{\prime} C^{\prime}} \cdot H(j \omega) ;
$$

where $R^{\prime}=R / R_{\mathrm{o}}$ and $C^{\prime}=C / C_{\mathrm{o}} ; R_{\mathrm{o}}$ and $C_{\mathrm{o}}$ are the input resistance and input capacitance (including stray contributions) of the detection instrument (such as an oscilloscope, etc.). Following Eq. (10), there is a zero point at $\omega=0$ and a pole at $\omega=1 /\left(R^{\prime} C^{\prime}\right)$, thus, there is a high cutoff frequency which can be determined to be $1 /\left(2 \pi R^{\prime} C^{\prime}\right)$. Generally, $R^{\prime} \approx R_{\mathrm{o}}$ and $C^{\prime} \approx C$ simply because $R \gg R_{\mathrm{o}}$ and $C \gg C_{\mathrm{o}}$. The oscilloscope we used had an input impedance of $10 \mathrm{M} \Omega / 15 \mathrm{pF}$, and a stray capacitance of $\sim 10 \mathrm{pF}$; and the ME laminate we used here had a capacitance of $C_{i}=4.86 \mathrm{nF}$. Thus, the cutoff frequency can be calculated as $3.2 \mathrm{~Hz}$, which coincides well with the experimental results shown in Fig. 2(a).

In practical ME sensor measurements, noise will always limit sensitivity. In a ME detection unit, we must consider two types of noise: intrinsic noise (Johnson, contact, etc.) and external noise (vibration, pyroelectric, etc.). We can refer all these noise sources to the input as $i_{n}(\omega)$, and redraw the equivalent circuit to include them, as given Fig. 1(d). In this case, the SNR can be expressed as

$$
\mathrm{SNR}=\frac{I(j \omega)}{i_{n}(\omega)}=\frac{\alpha_{0}(\omega) \cdot e^{-j \phi} \cdot j \omega \cdot H(j \omega)}{i_{n}(\omega)} .
$$

From Eq. (11), we can see that if $i_{n}(\omega)$ is less frequency dependent than the term $\alpha_{0}(\omega) \cdot e^{-j \phi} \cdot \omega$, then the SNR will increase with increasing frequency. Generally, at low frequencies and over short bandwidths, we can make an assumption that $\left|\alpha_{0}(\omega) \cdot e^{-j \phi}\right|$ is a constant.

We then constructed a ME sensor for testing, which was a longitudinal-transverse (L-T) mode laminate constructed from two Terfenol-D plates $\left(14 \times 6 \times 1.1 \mathrm{~mm}^{3}\right)$ and a single PZT one $\left(15 \times 6 \times 0.5 \mathrm{~mm}^{3}\right)$. Figure $2(\mathrm{~b})$ shows $\left|\alpha_{\mathrm{ME}}^{*}\right|$ for this $\mathrm{ME}$ sensor as a function of frequency over the range of $0.1 \mathrm{~Hz}<f<1000 \mathrm{~Hz}$. We can see in the range of $0.1 \mathrm{~Hz}$ $<f<100 \mathrm{~Hz}$ that $\left|\alpha_{\mathrm{ME}}^{*}\right|$ was nearly constant with a value of 
about $130 \mathrm{pC} /$ Oe. However, for $f>100 \mathrm{~Hz},\left|\alpha_{\mathrm{ME}}^{*}\right|$ decreased dramatically with increasing frequency. In our subsequent experiments, we focused on the frequency independent range, which is an appropriate range for detection of low frequency magnetic fields.

We also performed experiments to determine the signal linearity, the SNR, and the sensitivity limit (lowest signal detectable) for this L-T mode ME sensor. An ac magnetic field was generated by Helmoltz coils, which served as the source signal to be detected, and the output of the ME sensor was measured by a Keithley 428 low noise current amplifier. The equivalent model for the ME laminate and current amplifier is illustrated in Fig. 1(e). We can acquire the current gain $Z(\omega)$ of the circuit as

$$
Z(\omega)=\frac{R_{f}}{1+j \omega\left[R_{f} /(1+a)\right] C} \cdot H_{\mathrm{LP}}(j \omega) ;
$$

where $R_{f}$ is the feedback resistance, $a$ the open loop gain of the amplifier, and $H_{\mathrm{LP}}(j \omega)$ the transfer function of the low pass filter.

The signal output voltage $V_{s}$ and the output noise level $V_{n}$ are $^{10}$

$$
\begin{aligned}
& V_{s}=\alpha_{0}(\omega) \cdot e^{-j \phi} \cdot j \omega \cdot H(j \omega) \cdot Z(\omega) ; \\
& V_{n}=\left(\int_{f_{L}}^{f_{H}} i_{n}^{2} \cdot|Z(\omega)|^{2} d f\right)^{1 / 2} ;
\end{aligned}
$$

where $Z(\omega)$ is the current gain of the amplifier, and $f_{L}$ and $f_{H}$ are the lower and upper frequency limits of the bandwidth, respectively. Over the measurement bandwidth, Eq. (13a) can be simplified to

$$
V_{s}=\alpha_{0}(\omega) \cdot e^{-j \phi} \cdot j \omega \cdot H(j \omega) \cdot R_{f} \cdot H_{\mathrm{LP} 0} ;
$$

where $H_{\mathrm{LPO}}$ is the de gain of the low pass filter. The SNR then becomes

$$
\mathrm{SNR}=\frac{V_{s}}{V_{n}}=\alpha_{0}(\omega) \cdot e^{-j \phi} \cdot j \omega \cdot H(j \omega) \cdot \frac{R_{f} \cdot H_{\mathrm{LP} 0}}{\int_{f_{L}}^{f_{H}} i_{n}^{2} \cdot|Z|^{2} d f} .
$$

In Fig. 3(a), we can see that the ME sensor unit has good linearity at 1, 10, and $100 \mathrm{~Hz}$. These results agree with Eq. (14). It shows that $\left|\alpha_{0}(\omega) \cdot e^{-j \phi}\right|$ is (i) constant at low frequencies and (ii) independent of magnetic field in the low field range for low frequencies. In Fig. 3(b), we show the SNR and the sensitivity (i.e., lowest detectable magnetic field). In this figure, we can see that, with increasing frequency, the SNR increases and the lowest detectable signal decreases. These results agree with the predictions of Eq. (15) that the SNR decreases near linearly with decreasing frequency over
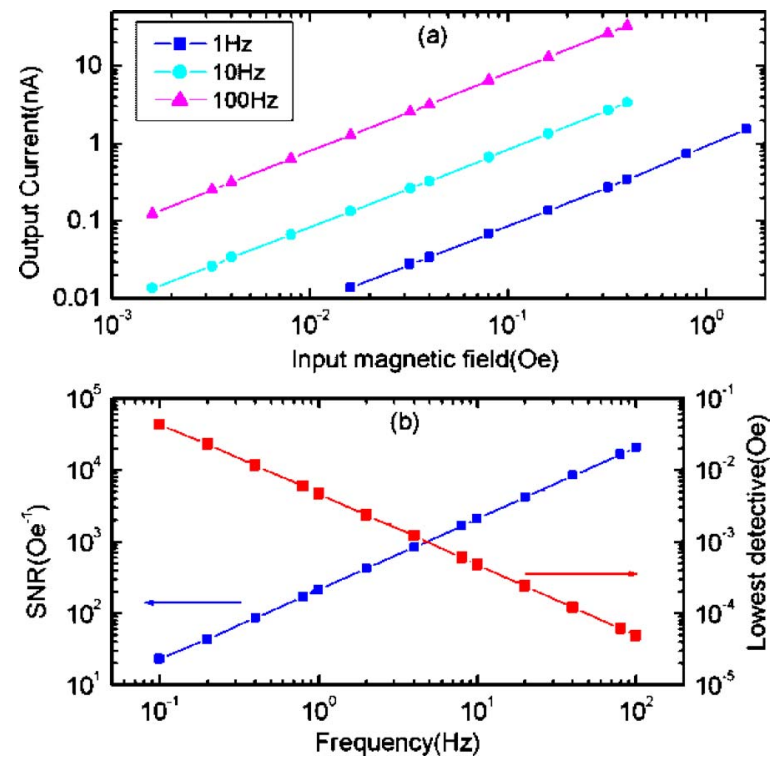

FIG. 3. (Color online) (a) Linearity of the ME sensor system at 1, 10, and $100 \mathrm{~Hz}$, and (b) SNR and lowest detective signal for L-T ME laminate.

the frequency range of $10^{-1} \mathrm{~Hz}<f<10^{2} \mathrm{~Hz}$. This result provides us helpful method of ME sensitivity estimation in the low frequency range. For example, if we know a ME laminte sensor has $1 \mathrm{nT}$ sensitivity at $1 \mathrm{~Hz}$, we can then estimate that the sensitivity of the ME sensor will become $0.1 \mathrm{nT}$ at $10 \mathrm{~Hz}$ and $10 \mathrm{nT}$ at $0.1 \mathrm{~Hz}$.

In summary, the SNR of a ME sensor unit has been predicted using a simple equivalent model. Experiments confirmed the predictions. The results provided insight into how to estimate the ME sensitivity in low frequency range through the one in a specific frequency, and how to enhance the sensitivity of real ME sensor units for the detection of small changes in quasistatic magnetic fields.

${ }^{1}$ M. Fiebig, J. Phys. D 38, 123 (2005)

${ }^{2}$ S. X. Dong, J. F. Li, and D. Viehland, Appl. Phys. Lett. 83, 2265 (2003). ${ }^{3}$ C. W. Nan, Phys. Rev. B 50, 6082 (1994).

${ }^{4}$ J. Ryu, C. Z. Carazo, K. Uchino, and H. E. Kim, Jpn. J. Appl. Phys., Part 1 40, 4948 (2001).

${ }^{5}$ S. X. Dong, J. Y. Zhai, F. M. Bai, J. F. Li, D. Viehland, and T. A. Lograsso, J. Appl. Phys. 97, 103902 (2005).

${ }^{6}$ J. Wang, J. B. Neaton, H. Zheng, V. Nagarajan, S. B. Ogale, B. Liu, D. Viehland, V. Vaithyanathan, D. G. Schlom, U. V. Waghmare, N. A. Spaldin, K. M. Rabe, M. Wuttig, and R. Ramesh, Science 299, 1719 (2003).

${ }^{7}$ G. Srinivasan, E. T. Rasmussen, and R. Hayes, Phys. Rev. B 67, 014418 (2003).

${ }^{8}$ M. I. Bichurin, D. A. Filippov, and V. M. Petrov, Phys. Rev. B 68, 132408 (2003).

${ }^{9}$ M. I. Bichurin, V. Petrov, and G. Srinivasan, Phys. Rev. B 68, 054402 (2003).

${ }^{10}$ S. Franco, Design with Operational Amplifiers and Analog Integrated Circuits (McGraw-Hill, New York, 2002), p. 318. 